\title{
Si-Doped InAs/GaAs Quantum-Dot Solar Cell With AlAs Cap Layers
}

\author{
Dongyoung Kim, Mingchu Tang, Jiang Wu, Sabina Hatch, Yurii Maidaniuk, Vitaliy Dorogan, \\ Yuriy I. Mazur, Gregory J. Salamo, and Huiyun Liu
}

\begin{abstract}
One of the requirements for strong subbandgap photon absorption in the quantum-dot intermediate-band solar cell (QD-IBSC) is the partial filling of the intermediate band. Studies have shown that the partial filling of the intermediate band can be achieved by introducing Si doping to the QDs. However, the existence of too many $\mathrm{Si}$ dopants leads to the formation of point defects and, hence, a reduction of photocurrent. In this study, the effect of Si doping on InAs/GaAs QD solar cells with AlAs cap layers is studied. The AlAs cap layers prevent the formation of the wetting layer during QD growth and reduce the Si doping density needed to achieve QD state filling. Furthermore, the passivation of defect states in the QD with moderate Si doping is demonstrated, which leads to an enhancement of the carrier lifetime in the QDs and, hence, the open-circuit voltage.
\end{abstract}

Index Terms-Intermediate-band solar cells (IBSCs), molecular beam epitaxy (MBE), quantum-dot (QD) solar cells.

\section{INTRODUCTION}

$\mathbf{S}$ INCE the concept of the intermediate-band solar cell (IBSC) was proposed in 1997, significant efforts have been made to realize IBSCs with efficiencies that exceed the Shockley-Queisser limit of 31\% [1]. The IBSC utilizes a collection of intermediate levels within the bandgap, called the intermediate band (IB), to absorb subbandgap energy photons. Its potential to exceed the Shockley-Queisser limit and reach the theoretical limit of $63.2 \%$ [2] arises from the additional photocurrent generated by the subbandgap photon absorption. One of the candidates for the high-efficiency IBSC is the quantumdot intermediate-band solar cell (QD-IBSC), which utilizes the discrete nature of the carrier density of states and the bandgap tunability of quantum dots (QDs). In a QD-IBSC, the IB is formed by the confined electron states in the QD array.

Manuscript received October 19, 2015; revised March 21, 2016; accepted March 22, 2016. Date of publication April 13, 2016; date of current version June 17, 2016. This work was supported by the Royal Society, the Defence Science Technology Laboratory, the U.K. Engineering and Physics Research Council under Grant EP/K029118/1, and the National Science Foundation of the U.S. under Grant DMR-1309989.

D. Kim, M. Tang, J. Wu, S. Hatch, and H. Liu are with the Department of Electronic and Electrical Engineering, University College London, London WC1E 7JE, U.K. (e-mail: d.kim@ucl.ac.uk; mingchu.tang.11@ucl.ac.uk; jiang. wu@ucl.ac.uk; s.hatch@ucl.ac.uk; huiyun.liu@ucl.ac.uk).

Y. Maidaniuk, V. Dorogan, Y. I. Mazur, and G. J. Salamo are with the Institute for Nanoscience and Engineering, University of Arkansas, Fayetteville, AK 72701 USA (e-mail: ymaidani@uark.edu; vdorogan@uark.edu; ymazur@ uark.edu; salamo@uark.edu).

Color versions of one or more of the figures in this paper are available online at http://ieeexplore.ieee.org.

Digital Object Identifier 10.1109/JPHOTOV.2016.2547581
However, over the last decade, several challenges in implementing high-efficiency QD-IBSCs have been identified. First, thermal decoupling between the IB and the conduction band (CB) of wetting layer (WL) needs to be achieved. Thermal coupling between the IB and CB enables the carriers to be thermally excited or relaxed between the IB and the CB [3]-[5]. This thermal process suppresses the second-photon absorption and lowers the effective bandgap energy of the solar cell leading to a reduced open-circuit voltage $\left(V_{\text {oc }}\right)$ [6], [7]. Tutu et al. demonstrated suppression of thermal escape of electrons in QDs by suppressing the formation of the WL via AlAs cap layer (CL) deposition [8], whereas Lam et al. reported reduced thermal coupling between the WL and QD states by introducing a potential barrier at the QD/WL interface via Si doping [9].

Second, the accumulated strain from the QDs leads to the formation of threading dislocations [10]-[12]. The strain-induced dislocations across the QD region result in a short minority carrier lifetime and hinders the stacking of QD layers that are needed to maximize the QD photon absorption [11]-[14]. Studies have shown that strain-induced dislocations can be minimized using a high-growth temperature GaAs spacer layer that separates each QD layer [10], [11]. Yang et al. demonstrated saturation of strain-induced dislocations in InAs QDs using Si doping [13].

Furthermore, the performance of IBSCs relies on an IB that is partially filled with electrons [15], [16]. For strong subbandgap photon absorption, the IB needs to have empty states to receive the electrons pumped from the VB and states filled with electrons to pump electrons to the CB. Luque and Martí proposed the use of doping as a method to achieve partial filling of the confined states in the IB [17]. It has also been demonstrated, by Martí et al. [18], that half-filling of the IB can be realized by doping the capping layers of QDs with $\mathrm{Si}$ in InAs/(Al, Ga)As quantum dot solar cells (QDSCs).

In this paper, the saturation of strain-induced dislocations and QD state filling are demonstrated by direct Si doping of InAs/GaAs QDSCs with AlAs CLs. Previously, we reported that the deposition of AlAs CLs on InAs QDs could suppress the formation of the WL [8]. Consequently, the effective bandgap of the QDSC was increased, which in turn led to the increase in the thermal activation energy and the $V_{\mathrm{OC}}$. In this study, in addition to the enhancement of the $V_{\mathrm{OC}}$ by applying AlAs CLs, a further increase in the $V_{\mathrm{OC}}$ by $\sim 44 \mathrm{mV}$ is observed by passivating the defect states with moderate Si doping (6 e/dot). In addition, QD state filling, which is essential for strong two-photon absorption, is observed with significantly lower $\mathrm{Si}$ doping densities when compared with that of our previous study [9]. 


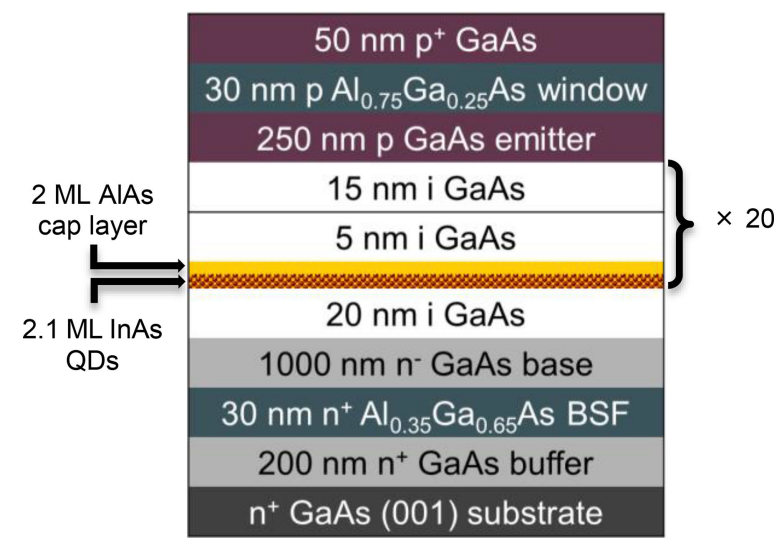

Fig. 1. Structure of the Si-doped $(0,6,12,18$ e/dot) InAs/GaAs QDSCs with AlAs CLs.

\section{EXPERIMENTAL DETAILS}

The InAs/GaAs QDSC samples with AlAs CLs were grown by a solid-source molecular beam epitaxy (MBE) on $\mathrm{n}^{+} \mathrm{GaAs}$ $\left(\begin{array}{lll}1 & 0 & 0\end{array}\right)$ substrates. As shown in Fig. 1, all SCs were grown with the same p-i-n structure that consists of a $200-\mathrm{nm}$ GaAs buffer layer with Si doping density of $1 \times 10^{18} \mathrm{~cm}^{-3}, 30-\mathrm{nm}$ $\mathrm{Al}_{0.35} \mathrm{Ga}_{0.65}$ As back surface field with Si doping density of 1 $\times 10^{18} \mathrm{~cm}^{-3}, 1000-\mathrm{nm}$ GaAs base with Si doping density of 1 $\times 10^{17} \mathrm{~cm}^{-3}, 250-\mathrm{nm}$ GaAs emitter with Be doping density of $2 \times 10^{18} \mathrm{~cm}^{-3}, 100$-nm GaAs emitter with Be doping density of $5 \times 10^{18} \mathrm{~cm}^{-3}, 30-\mathrm{nm} \mathrm{Al} \mathrm{Al}_{0.75} \mathrm{Ga}_{0.25}$ As window layer with Be doping density of $2 \times 10^{18} \mathrm{~cm}^{-3}$, and 50-nm GaAs contact layer with Be doping density of $1 \times 10^{19} \mathrm{~cm}^{-3}$. The intrinsic region of the SCs consists of 20 stacks of 2.1 monolayer (ML) InAs QDs with two ML AlAs CLs separated by a 20-nm GaAs spacer. The QDs were grown by the Stranski-Krastanov mode at substrate temperature of $\sim 500{ }^{\circ} \mathrm{C}$. High-growth temperature GaAs spacer layers were applied during the growth of QDs to suppress the formation of dislocations [10], [11], [19]. The QDSCs underwent direct Si doping with four different doping densities of $0,6,12$, and 18 e/dot.

Prior to device fabrication, the samples were ultrasonicated first in acetone and then in isopropanol for 10 min each. For the surface oxide removal, the samples were immersed in dilute ammonia solution (1:19) for $30 \mathrm{~s}$. A Au-Zn alloy (95\% Au, $5 \% \mathrm{Zn}$ ) was thermally evaporated to form a ( 200-nm-thick) grid-pattern p-type electrode using a metal shadow mask. For the n-type electrode, 10-nm Ni, 100-nm Au-Ge (88\% Au, $12 \%$ $\mathrm{Ge}$ ), 30-nm Ni, and 200-nm Au were thermally evaporated onto the entire back surface and thermally annealed at $400^{\circ} \mathrm{C}$ for $60 \mathrm{~s}$ in forming gas. No antireflective coating or surface passivation was applied to these SCs.

A Veeco Nanoscope $\mathrm{V}$ atomic force microscope (AFM) was used to characterize the morphology of an uncapped QD layer. Temperature-dependent and power-dependent photoluminescence (PL) measurements were performed using 532-nm excitation from a diode-pumped solid-state laser. The sample temperature between 10 and $300 \mathrm{~K}$ was controlled using a Hecooled cryostat during the PL measurements. The transient PL measurements were performed with 2-ps pulses at excitation

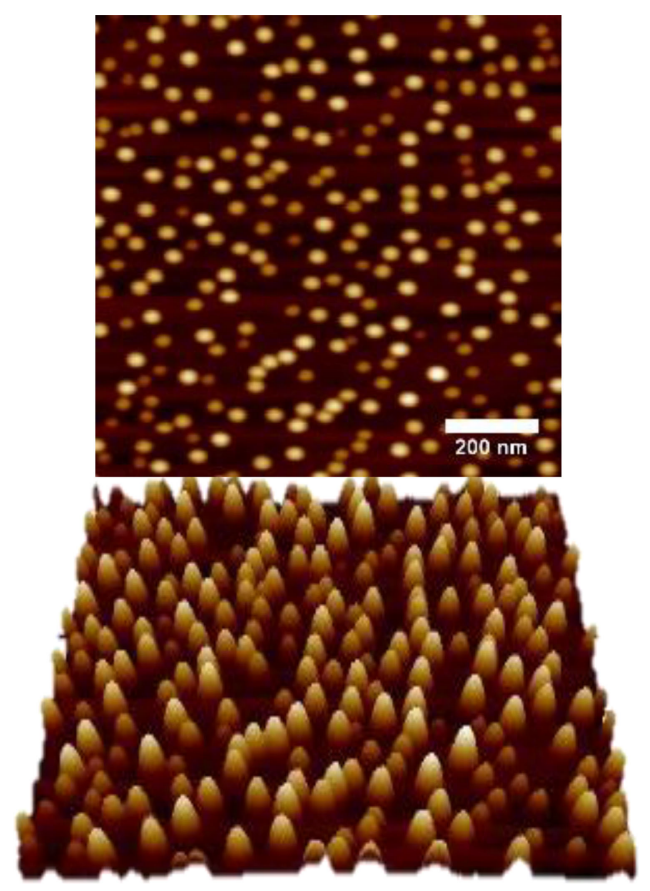

Fig. 2. AFM images of InAs QDs grown on GaAs in 2-D (top) and 3-D (bottom).

of $750 \mathrm{~nm}$ from a mode-locked Ti:sapphire laser that produces an optical pulse train at $76 \mathrm{MHz}$. In addition, a Hamamatsu Synchroscan streak camera C5680 with an infrared enhanced S1 cathode was used for the transient PL signal detection. Current density versus voltage $(J-V)$ characteristics were obtained by using an LOT calibrated solar simulator with a xenon lamp under 1-sun air mass (AM) $1.5 \mathrm{G}$ illumination at room temperature. A four-point probe station was used to connect devices to a Keithly 2400 sourcemeter that outputs the data to ReRa Tracer 3 software. Photocurrent measurements were performed with a halogen lamp chopped to a frequency of $188 \mathrm{~Hz}$ through a Newport monochromator. The monochromatic beam was calibrated with a silicon photodiode, and the data were analyzed with ReRa Photor QE 3.1 software to obtain the external quantum efficiency (EQE) at room temperature.

\section{RESUlTS AND DISCUSSION}

AFM was used to analyze the morphology of InAs QDs on GaAs, as shown in Fig. 2. The average diameter of the QDs was $\sim 30 \mathrm{~nm}$, with an average height of $\sim 5 \mathrm{~nm}$. No large defective clusters were observed, which indicates a high structural quality [20]. The dot density was estimated to be $\sim 2.3 \times 10^{10} \mathrm{~cm}^{-2}$.

The optical properties of the Si-doped InAs/GaAs QDs with AlAs CLs were compared. Fig. 3(a) shows the normalized PL spectra for the QDSCs at $10 \mathrm{~K}$. The PL spectrum of the undoped QDSC ( 0 e/dot) shows two peaks at $\sim 830$ and $\sim 1050 \mathrm{~nm}$, originating from the GaAs and InAs QD ground state, respectively. The spectra display additional QD emission peaks (950$1100 \mathrm{~nm}$ ) for the samples with higher Si doping. This can be explained by the emissions related to excited state transitions observed at shorter wavelengths alongside the ground state 


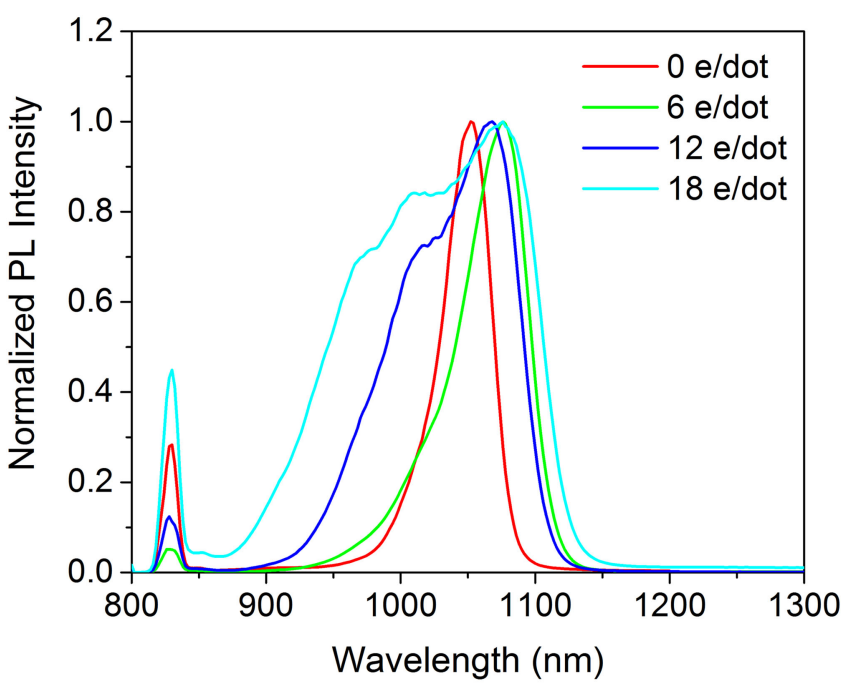

(a)
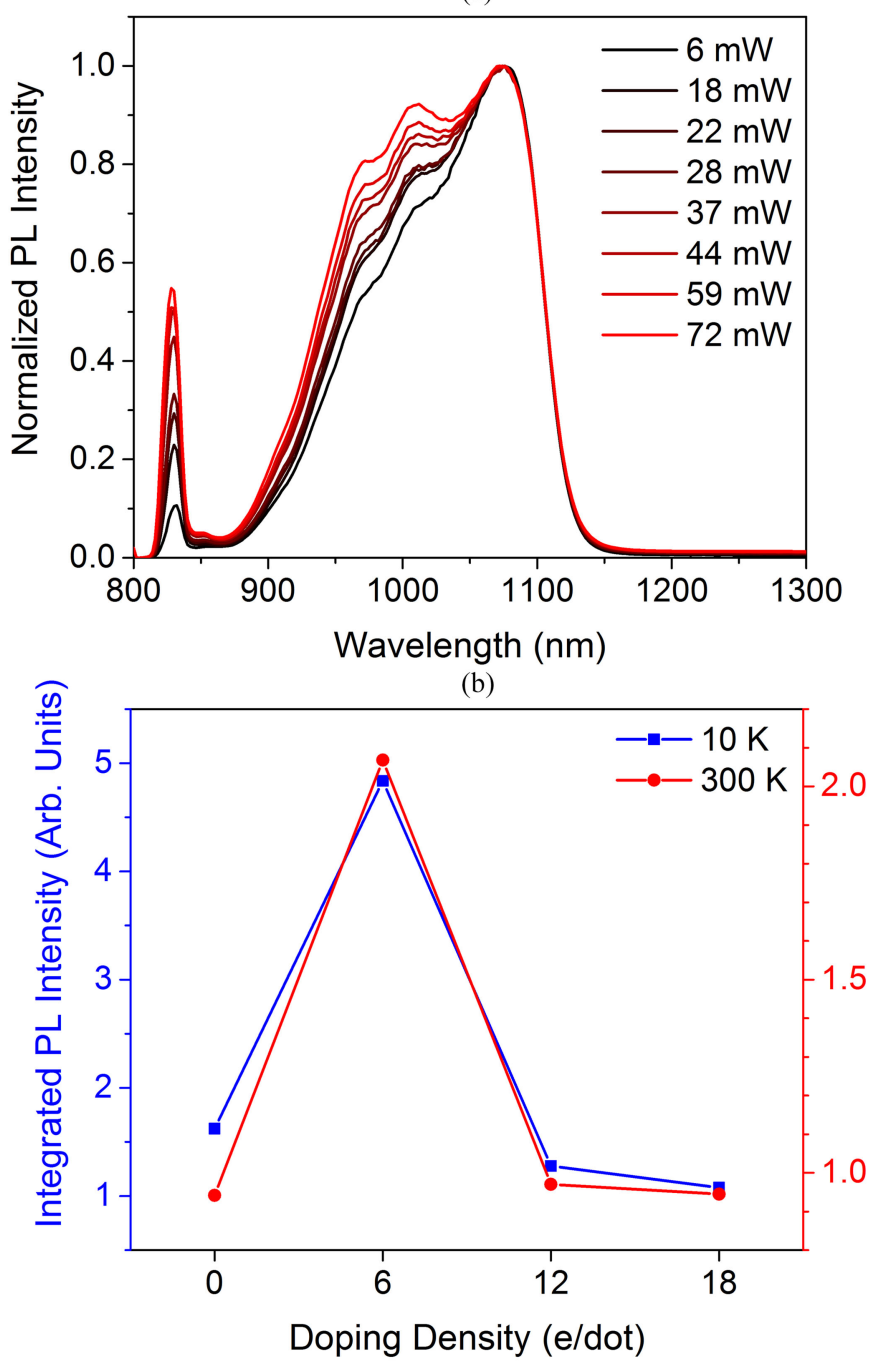

(c)

Fig. 3. (a) Normalized PL spectra of Si-doped (0, 6, 12, 18 e/dot) QDSCs with AlAs CL measured at $10 \mathrm{~K}\left(P_{\mathrm{ex}}=37 \mathrm{~mW}\right)$. (b) Normalized power-dependent PL spectra for 18 e/dot QDSC at $10 \mathrm{~K}$. (c) Integrated PL intensity versus Si doping density at $10 \mathrm{~K}\left(I_{\mathrm{ex}}=386 \mathrm{~W} / \mathrm{cm}^{2}, \lambda_{\mathrm{ex}}=532 \mathrm{~nm}\right)$ and $300 \mathrm{~K}$ $\left(I_{\mathrm{ex}}=459 \mathrm{~W} / \mathrm{cm}^{2}, \lambda_{\mathrm{ex}}=635 \mathrm{~nm}\right)$. emission [21]. Although the higher energy emission peaks can originate from the phonon bottleneck, segregated inhomogeneous broadening, and state filling, it is possible to distinguish them by performing a power-dependent PL study. Fig. 3(b) shows the normalized power-dependent PL spectra for the QDSC with Si doping density of 18 e/dot. It shows that with decreasing laser excitation power, the relative magnitudes of the peaks observed between 950 and $1025 \mathrm{~nm}$ decrease, when compared with the peak at $\sim 1080 \mathrm{~nm}$. This indicates that the emission peaks are caused by the state filling effect, where the lowest energy peak is the ground state, and the higher energy peaks are the excited states [22], [23]. The trend that shows excited state emissions become more prominent with higher Si doping density in Fig. 3(a) indicates that the doping results in state filling effect. In our previous study [9], even though the dopants were directly supplied to the QDs, some dopants remained in the WL and led to a potential barrier forming between the WL and QDs. In contrast, this work prevents the formation of WLs by applying AlAs CLs on QDs during the MBE growth [8]. In this manner, the Si doping is concentrated inside the QDs rather than the WL and contributes to state filling. This explains why the PL peaks from WL emissions that were observed in our previous study with the same Si doping densities [9] are no longer visible, and instead, additional peaks from state filling are observed in Fig. 3(a).

Fig. 3(c) shows the integrated PL intensity as a function of $\mathrm{Si}$ doping density at 10 and $300 \mathrm{~K}$. The QDSC with doping density of 6 e/dot exhibits the strongest PL peak intensity. The enhancement of the PL intensity can be attributed to the passivation of the defect states by Si doping and leads to the suppression of nonradiative recombination [24], [25]. However, Fig. 3(c) shows that any further increase in the Si doping level results in a dramatic decrease in the PL intensity. This could be attributed to excess $\mathrm{Si}$ atoms that can introduce nonradiative recombination centers [13]. Another possibility is that Si doping introduces additional carriers, which lead to higher Auger recombination rate [26], [27].

In order to provide further evidence of the QD state filling and the passivation of defect states achieved by Si doping, a time-resolved PL study was performed for the QDCSs at $10 \mathrm{~K}$. Fig. 4 shows that the carrier lifetime in the region of 875$925 \mathrm{~nm}$ increases with increasing Si doping density. This can be linked to the state filling effect observed from the steady-state PL measurements in Fig. 3(a). The appearance of the higher energy QD emission peaks with higher Si doping densities in Fig. 3(a) indicates that the additional electrons supplied by the Si dopants are used to fill the QD states. With more QD states filled with electrons, fewer available decay channels exist for the carriers, which in turn results in longer carrier lifetimes. In contrast, the carrier lifetime of the QD ground state $(1050-1100 \mathrm{~nm})$ in Fig. 4 shows that increasing the Si doping density leads to an initial increase with moderate doping density (6 e/dot), followed by a significant drop in the carrier lifetime with higher doping densities $(12,18 \mathrm{e} / \mathrm{dot})$. This trend is similar to that observed in the integrated PL intensity measurements, as shown in Fig. 3(c). Therefore, it can be inferred that the initial increase in the carrier lifetime with doping density of 6 e/dot originates 


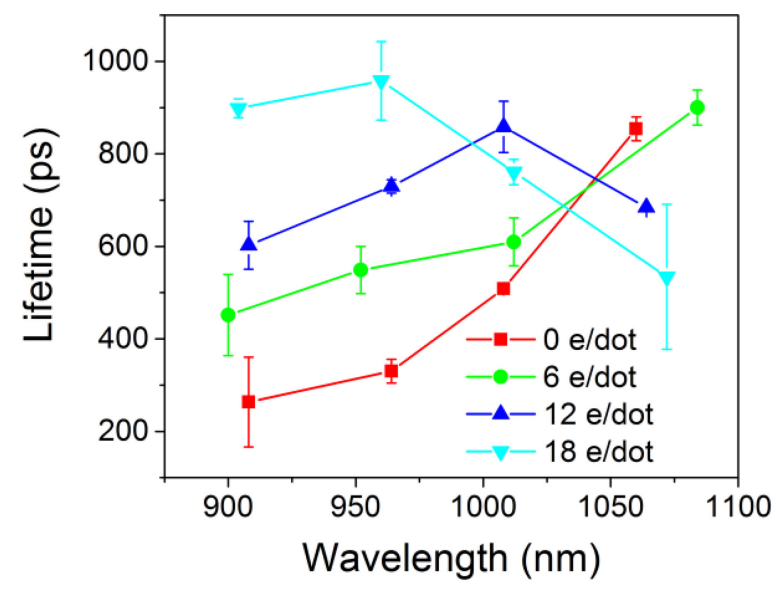

Fig. 4. Carrier lifetime versus wavelength obtained from the transient PL spectra of the Si-doped QDSCs with AlAs CLs at $10 \mathrm{~K}\left(\lambda_{\mathrm{ex}}=750 \mathrm{~nm}\right)$.

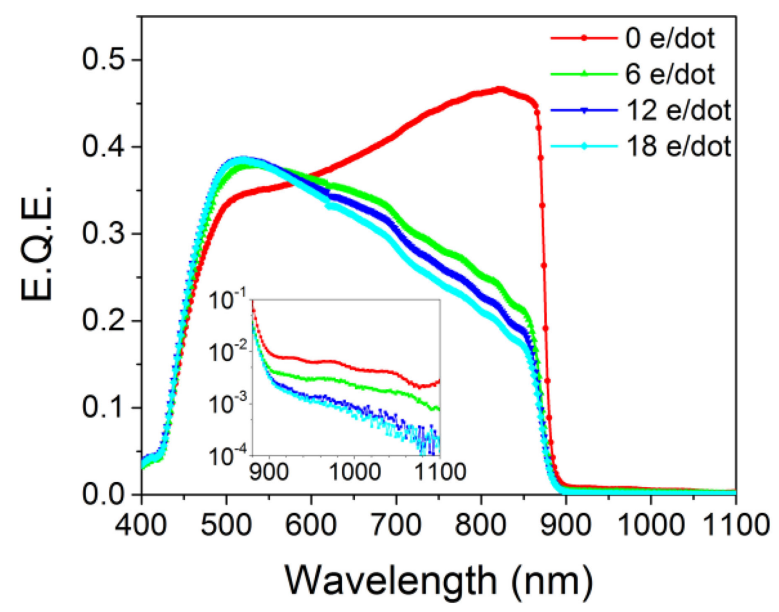

Fig. 5. Linear-scale EQE spectra of Si-doped $(0,6,12,18$ e/dot) QDSCs with AlAs CLs. Inset shows semilog scale of subbandgap EQE.

from the passivated defect states. However, once all defect states are filled, further addition of $\mathrm{Si}$ atoms leads to the formation of nonradiative recombination centers, and/or increase Auger scattering rate, which decreases the carrier lifetime.

The EQE spectra of the Si-doped QDSCs with AlAs CLs are presented in Fig. 5. For all doped samples, there is a drop in EQE at $\sim 870 \mathrm{~nm}$, which corresponds to the bandgap of GaAs. At the higher wavelengths, flat spectral responses without a WL peak $(\sim 915 \mathrm{~nm})$ are observed. The subbandgap EQE spectra shown in the inset of Fig. 5 depicts decreasing EQE contribution from the QDs with increasing doping density. This is primarily attributed to the extra electrons introduced by Si doping filling the $\mathrm{CB}$ of the QDs, which decreases the probability of the VB to IB transition. As a result, the absorption from the QDs is weakened [13]. Another possibility for the reduced EQE is the suppression of thermal escape of photoexcited carriers due to the formation of a potential barrier at QD/WL interface by $\mathrm{Si}$ doping, as reported in our previous study [9]. However, with the AlAs CL, it is expected that the formation of such a potential barrier be minimized. It can be also noted from Fig. 5 that the supra-bandgap (400-870 $\mathrm{nm}$ ) absorption of all QDSCs with

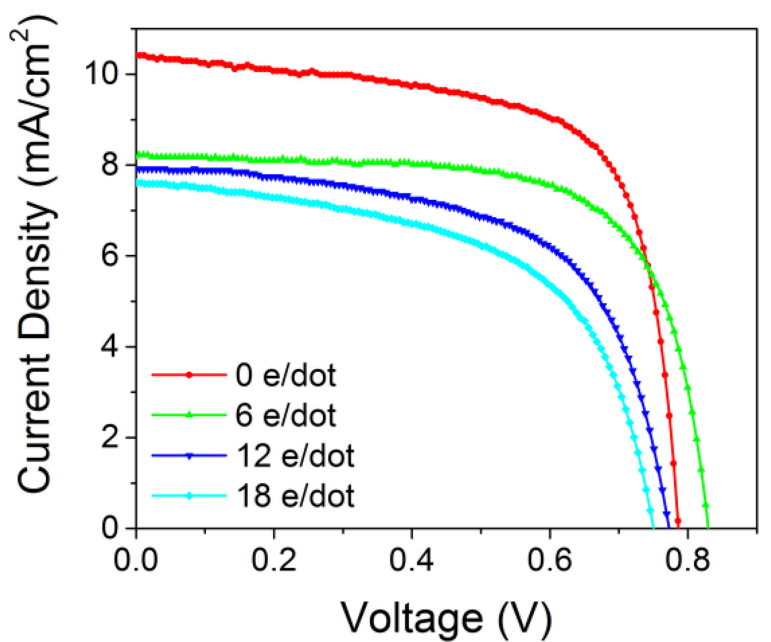

Fig. 6. Current density versus voltage behavior of Si-doped QDSCs with AlAs CLs under 1-sun (AM 1.5G) illumination.

TABLE I

Current Density, Open-CirCuit Voltage, Fill Factor, AND EFFiciency MEASURED FROM SI-DOPED QDSCS WITH ALAS CLS

\begin{tabular}{lcccc}
\hline \hline Device & $J_{\mathrm{SC}}\left(\mathrm{mA} / \mathrm{cm}^{2}\right)$ & $V_{\mathrm{OC}}(\mathrm{V})$ & $\mathrm{FF}(\%)$ & $\eta(\%)$ \\
\hline $0 \mathrm{e} / \mathrm{dot}$ & 10.41 & 785.76 & 68.49 & 5.60 \\
$6 \mathrm{e} / \mathrm{dot}$ & 8.19 & 829.34 & 69.04 & 4.69 \\
$12 \mathrm{e} / \mathrm{dot}$ & 7.91 & 772.40 & 60.69 & 3.71 \\
$18 \mathrm{e} / \mathrm{dot}$ & 7.61 & 749.82 & 56.98 & 3.25 \\
\hline \hline
\end{tabular}

Si doping is significantly reduced when compared with that of the undoped QDSC. This can be attributed to the decrease in depletion region after $\mathrm{Si}$ doping, which reduces the effective absorption area. At the same time, the reduction in the suprabandgap EQE could be linked to the introduction of Si dopants. The Si dopants can substitute the Ga and As sites or exist as interstitials [28], [29]. This leads to the formation of point defects and, hence, a reduction in the EQE response.

The $J-V$ characteristics of the QDSCs in Fig. 6 and Table I show a clear dependence on the Si-doping density. First, all Sidoped samples display a significant drop in the current density when compared with the undoped sample. This can be related to the reduction in the $\mathrm{EQE}$ contribution from the supra-bandgap region observed in Fig. 5. In other words, the decrease in the depletion region width caused by the introduction of Si dopants results in a reduction in the effective area for absorption and, hence, a decrease in the current density. Second, a further gradual decrease in the current density with increasing doping is observed among the doped QDSCs. This is likely to be due to the point defects formed by $\mathrm{Si}$ dopants substituting $\mathrm{Ga}$ and As or existing as interstitials [28], [29]. The defect states induced by Si doping increase Shockley-Read-Hall recombination decrease the minority carrier lifetime and, consequently, lower the current density. Although Si doping has a negative impact in the current density, an enhancement of the $V_{\mathrm{OC}}$ is observed with moderate doping density. The $V_{\mathrm{OC}}$ increases from $0.783 \mathrm{~V}$ for the 0 e/dot QDSC to $0.826 \mathrm{~V}$ for the 6 e/dot QDSC. This agrees well with the trend observed in the PL intensity measurements 
shown in Fig. 3(c). Both the PL intensity and the $V_{\mathrm{OC}}$ reach their maxima at $6 \mathrm{e} / \mathrm{dot}$, which is likely to be due to the moderate Si doping passivating the defect states. However, further increase in the $\mathrm{Si}$ doping leads to a gradual decrease in the $V_{\mathrm{OC}}$. This corresponds well with the decreasing PL intensity in Fig. 3(c) that originates from the formation of nonradiative recombination centers due to the excessive concentration of $\mathrm{Si}$ atoms.

\section{CONCLUSION}

In conclusion, an improvement in the $V_{\mathrm{OC}}(\sim 44 \mathrm{mV})$ has been achieved by introducing direct $\mathrm{Si}$ doping to QDs with AlAs CLs. The increase in the $V_{\mathrm{OC}}$ is attributed to the passivation of the defect states with moderate Si doping ( 6 e/dot), along with the reduced CB-IB thermal coupling with AlAs CLs. In addition, with the AlAs CLs, the QD state filling effect is observed with low Si doping densities. The factors that contributed to the decrease in the supra-bandgap EQE and the photocurrent after Si doping, such as the decrease in depletion region and the formation of point defects, should be taken into account when designing future Si-doped QDSCs. Nonetheless, the results presented here hold some promise for overcoming some of the main challenges in implementing QD-IBSCs with the efficiencies close to that of the theoretical model of the IBSC.

\section{REFERENCES}

[1] W. Shockley and H. J. Queisser, "Detailed balance limit of efficiency of p-n junction solar cells," J. Appl. Phys., vol. 32, no. 3, pp. 510-519, Mar. 1961.

[2] A. Luque, A. Martí, and C. Stanley, "Understanding intermediate-band solar cells," Nat. Photon., vol. 6, no. 3, pp. 146-152, Mar. 2012.

[3] S. Sanguinetti et al., "Carrier thermal escape and retrapping in selfassembled quantum dots," Phys. Rev. B, vol. 60, no. 11, pp. 8276-8283, Sep. 1999.

[4] E. Antolín et al., "Reducing carrier escape in the InAs/GaAs quantum dot intermediate band solar cell," J. Appl. Phys., vol. 108, no. 6, art. no. 064513, Sep. 2010.

[5] A. Luque and A. Martí, "The intermediate band solar cell: Progress toward the realization of an attractive concept," Adv. Mater., vol. 22, no. 2, pp. 160-174, Nov. 2010.

[6] C. G. Bailey, D. V. Forbes, R. P. Raffaelle, and S. M. Hubbard, "Near 1 V open circuit voltage InAs/GaAs quantum dot solar cells," Appl. Phys. Lett., vol. 98, no. 16, art. no. 163105, Apr. 2011.

[7] A. Mellor, A. Luque, I. Tobías, and A. Martí, "Realistic detailed balance study of the quantum efficiency of quantum dot solar cells," Adv. Funct. Mater, vol. 24, no. 3, pp. 339-345, Jan. 2014.

[8] F. K. Tutu et al., "InAs/GaAs quantum dot solar cell with an AlAs cap layer," Appl. Phys. Lett., vol. 102, no. 16, art. no. 163907, Apr. 2013.

[9] P. Lam et al., "Voltage recovery in charged InAs/GaAs quantum dot solar cells," Nano Energy, vol. 6, pp. 159-166, May 2014.

[10] H. Y. Liu et al., "Improved performance of $1.3 \mu \mathrm{m}$ multilayer InAs quantum-dot lasers using a high-growth-temperature GaAs spacer layer," Appl. Phys. Lett., vol. 85, no. 5, pp. 704-706, Aug. 2004.

[11] F. K. Tutu et al., "Improved performance of multilayer InAs/GaAs quantum-dot solar cells using a high-growth-temperature GaAs spacer layer," J. Appl. Phys., vol. 111, no. 4, art. no. 046101, Feb. 2012.

[12] A. Martí et al., "Emitter degradation in quantum dot intermediate band solar cells," Appl. Phys. Lett., vol. 90, no. 23, art. no. 233510, Jun. 2007.

[13] X. Yang et al., "Improved efficiency of InAs/GaAs quantum dots solar cells by Si-doping," Sol. Energy Mater. Sol. Cells, vol. 113, pp. 144-147, Jun. 2013

[14] T. Sugaya et al., "Multi-stacked quantum dot solar cells fabricated by intermittent deposition of InGaAs," Sol. Energy Mater. Sol. Cells, vol. 95, no. 1, pp. 163-166, Jan. 2011.
[15] A. Martí, L. Cuadra, and A. Luque, "Partial filling of a quantum dot intermediate band for solar cells," IEEE Trans. Electron Devices, vol. 48, no. 10, pp. 2394-2399, Oct. 2001.

[16] A. Martí et al., "Novel semiconductor solar cell structures: The quantum dot intermediate band solar cell," Thin Solid Films, vols. 511/512, pp. 638-644, Jul. 2006.

[17] A. Luque and A. Martí, "A metallic intermediate band high efficiency solar cell," Prog. Photovoltaics, Res. Appl., vol. 9, no. 2, pp. 73-86, Mar. 2001.

[18] A. Martí et al., "Production of photocurrent due to intermediate-toconduction-band transitions: a demonstration of a key operating principle of the intermediate-band solar cell," Phys. Rev. Lett., vol. 97, no. 24, art. no. 247701, Dec. 2006.

[19] H. Y. Liu et al., "p-doped 1.3 $\mu \mathrm{mInAs} / \mathrm{GaAs}$ quantum-dot laser with a low threshold current density and high differential efficiency," Appl. Phys. Lett., vol. 89, no. 7, art. no. 073113, Aug. 2006.

[20] H. Y. Liu et al., "Influences of the spacer layer growth temperature on multilayer InAs/GaAs quantum dot structures," J. Appl. Phys., vol. 96, no. 4, pp. 1988-1992, Aug. 2004.

[21] M. V. Marquezini et al., "Study of temperature-dependent exciton dynamics in a single quantum well with self-assembled islands," Surf. Sci., vols. 361/362, pp. 810-813, Jul. 1996.

[22] K. Brunner et al., "Photoluminescence from a single GaAs/AlGaAs quantum dot," Phys. Rev. Lett., vol. 69, no. 22, pp. 3216-3219, Nov. 1992.

[23] Z. M. Wang, Self-Assembled Quantum Dots. New York, NY, USA Springer, 2007.

[24] K. A. Sablon et al., "Strong enhancement of solar cell efficiency due to quantum dots with built-in charge," Nano Lett., vol. 11, no. 6, pp. 23112317, 2011.

[25] T. Inoue, S. Kido, K. Sasayama, T. Kita, and O. Wada, "Impurity doping in self-assembled InAs/GaAs quantum dots by selection of growth steps," J. Appl. Phys., vol. 108, no. 6, art. no. 063524, Sep. 2010.

[26] D. Morris, N. Perret, and S. Fafard, "Carrier energy relaxation by means of Auger processes in InAs/GaAs self-assembled quantum dots," Appl. Phys. Lett., vol. 75, no. 23, pp. 3593-3595, Dec. 1999.

[27] J. Siegert, S. Marcinkevičius, and Q. X. Zhao, "Carrier dynamics in modulation-doped InAs/GaAs quantum dots," Phys. Rev. B, vol. 72, no. 8, art. no. 085316, Aug. 2005.

[28] D. T. J. Hurle, "A comprehensive thermodynamic analysis of native point defect and dopant solubilities in gallium arsenide," J. Appl. Phys., vol. 85, no. 10, pp. 6957-7022, May 1999.

[29] D. T. J. Hurle, "A thermodynamic analysis of native point defect and dopant solubilities in zinc-blende III-V semiconductors," J. Appl. Phys. vol. 107, no. 12, art. no. 121301, Jun. 2010.

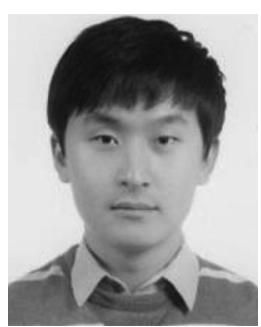

Dongyoung Kim was born in Daegu, South Korea. He received the M.Eng. degree in electronic and electrical engineering from University College London, London, U.K., in 2013, where he is currently working toward the Ph.D. degree with the Molecular Beam Epitaxy Research Group.

His current research interests include III-V quantum-dot solar cells and nanowires.

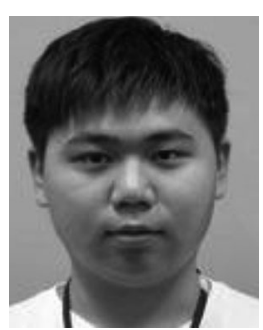

Mingchu Tang received the B.Eng. degree in electronic and electrical engineering from the University of Sussex, Brighton, U.K., in 2011. He is currently working toward the Ph.D. degree with University College London, London, U.K.

His current research interests include III-V quantum-dot materials and devices and molecular beam epitaxy growth. 


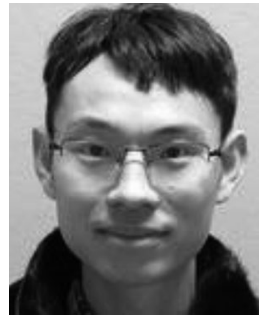

Jiang Wu received the B.S. degree from the University of Electronic Science and Technology of China, Chengdu, China, and the M.S. and Ph.D. degrees from the University of Arkansas-Fayetteville, Fayetteville, AR, USA, in 2008 and 2011, respectively.

$\mathrm{He}$ is currently a Lecturer with the Department of Electrical and Electronic Engineering, University College London, London, U.K. His research interests include molecular beam epitaxy growth and optoelectronic devices, including photovoltaic cells, lasers, and photodetectors.

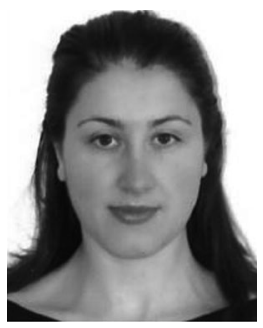

Sabina Hatch received the M.Sc. degree in physics from Bristol University, Bristol, U.K., and the Ph.D. degree in material science from the Queen Mary University of London, London, U.K., in 2013.

She is currently a Postdoctoral Research Associate with the Department of Electrical and Electronic Engineering, University College London. Her research interests include the fabrication of purely inorganic and inorganic-organic hybrid optoelectronic devices using low-dimensional III-V structures (i.e., quantum well, quantum dots, and quantum nanowires)

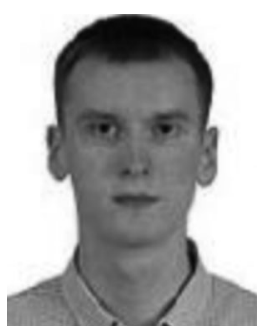

Yurii Maidaniuk received the B.S. and M.S. degrees in physics from the Physics Department, Taras Shevchenko National University of Kiev, Kiev, Ukraine, in 2011 and 2013, respectively. He is currently working toward the Ph.D. degree with the University of Arkansas, Fayetteville, AR, USA.

His research interests include the optoelectronic properties of semiconductor nanostructures.

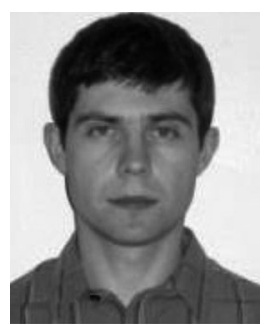

Vitaliy Dorogan received the B.S. and M.S. degrees in physics from the Physics Department, Uzhgorod State University, Uzhgorod, Ukraine, in 1999 and 2000, respectively, and the Ph.D. degree in microelectronics-photonics from the University of Arkansas, Fayetteville, AR, USA, in 2011.

$\mathrm{He}$ is currently a Research Assistant Professor with the Institute for Nanoscience and Engineering, University of Arkansas. His current research interests include the spectroscopic study of semiconductor nanostructures.

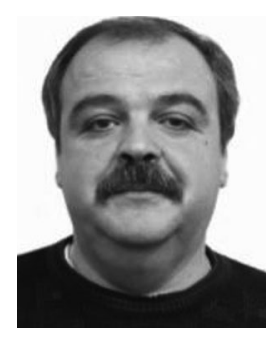

Yuriy I. Mazur received the M.Sc. degree in solidstate physics from the Moscow Institute of Physics and Engineering, Moscow, USSR, in 1978 and the $\mathrm{Ph} . \mathrm{D}$. degree in solid-state physics from the Institute of General Physics, Moscow, in 1983.

He worked with the Institute of Semiconductors Physics, National Academy of Sciences, Kiev, Ukraine, and Max-Born-Institute Berlin, Germany, as a Senior Research Associate and Visiting Scientist during 1982-2000. He joined the Department of Physics, University of Arkansas, Fayetteville, AR, USA, in 2001, where he is currently a Research Professor with the Institute for Nanoscience and Engineering. His research interests include the physics of magnetic phenomena and disordering in semimagnetic semiconductors, physics of 2-D electron (hole) states, and excitons in III-V semiconductor heterostructures and quantum dots.

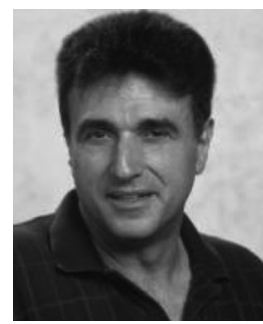

Gregory J. Salamo received the Ph.D. degree in physics from the City University of New York, New York, NY, USA, in 1973. He was an Intern Student with Bell Laboratories, Murray Hill, NJ, USA.

He was a Postdoctoral Researcher with the Institute of Optics, University of Rochester, Rochester, NY. In 1975, he joined the faculty of the University of Arkansas, Fayetteville, AR, USA, where he is currently a Distinguished Professor of physics. He is the Co-Director of the National Science Foundation (NSF) Materials Research Science and Engineering Center on the physics of semiconductor nanostructures. He has published more than 300 papers in referred journals, given numerous contributed and invited talks, and contributed several book chapters. He has carried out research in the areas of optical communications, optical image processing, and the optical properties of semiconductors. He is involved in the development of interdisciplinary research and education through the establishment of a new degree program to provide greater career opportunities for students and faculty. He is also involved in the development of an educational course and laboratory in nanotechnology and a GK-12 NSF program for middle school students. His current research interests include growing III-V semiconductors and ferroelectrics using molecular beam epitaxy and scanning tunneling microscopy.

Dr. Salamo is a Fellow of the Optical Society of America.

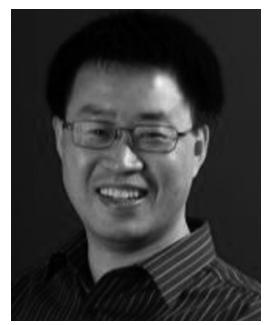

Huiyun Liu received the Ph.D. degree in semiconductor science from the Institute of Semiconductors, Chinese Academy of Sciences, Beijing, China

He then joined the EPSRC National Centre for III-V Technologies, University of Sheffield, Sheffield, U.K., in August 2001. In 2007, he received a Royal Society University Research Fellowship and joined the Department of Electronic and Electrical Engineering, University College London, London, U.K., where he is currently a Professor of semiconductor photonics. He has been working on the development of III-V compound materials and devices. His current research interests include the nanometer-scale engineering of low-dimensional semiconductor structures (such as quantum dots, nanowires, and quantum wells) by using molecular beam epitaxy and the development of novel optoelectronic devices including solar cells, lasers, detectors, and modulators by developing novel device process techniques. 